ORIGINAL ARTICLE

\title{
Assessment of ventilatory thresholds during graded and maximal exercise test using time varying analysis of respiratory sinus arrhythmia
}

\author{
G Blain, O Meste, T Bouchard, S Bermon
}

Br J Sports Med 2005;39:448-452. doi: 10.1136/bjsm.2004.014134

See end of article for authors' affiliations

......................

Correspondence to:

Gregory Blain, Universite

de Toulon-Var,

Département Érgonomie

Sportive et Performances,

Avenue Valombrose, NICE

cedex 206107 , France;

blain@unice.fr

Accepted

7 September 2004
Objective: To test whether ventilatory thresholds, measured during an exercise test, could be assessed using time varying analysis of respiratory sinus arrhythmia frequency $\left(f_{\mathrm{RSA}}\right)$.

Methods: Fourteen sedentary subjects and 12 endurance athletes performed a graded and maximal exercise test on a cycle ergometer: initial load $75 \mathrm{~W}$ (sedentary subjects) and $150 \mathrm{~W}$ (athletes), increments $37.5 \mathrm{~W} / 2 \mathrm{~min}$. $f_{\mathrm{RSA}}$ was extracted from heart period series using an evolutive model. First $\left(T_{\mathrm{V} 1}\right)$ and second $\left(T_{\mathrm{V} 2}\right)$ ventilatory thresholds were determined from the time course curves of ventilation and ventilatory equivalents for $\mathrm{O}_{2}$ and $\mathrm{CO}_{2}$.

Results: $f_{\text {RSA }}$ was accurately extracted from all recordings and positively correlated to respiratory frequency $(r=0.96(0.03), p<0.01)$. In 21 of the 26 subjects, two successive non-linear increases were determined in $f_{R S A}$, defining the first $\left(T_{R S A}\right)$ and second $\left(T_{R S A 2}\right) f_{R S A}$ thresholds. When expressed as a function of power, $T_{R S A 1}$ and $T_{R S A 2}$ were not significantly different from and closely linked to $T_{V 1}(r=0.99$, $p<0.001)$ and $T_{V 2}(r=0.99, p<0.001)$, respectively. In the five remaining subjects, only one non-linear increase was observed close to $T_{V 2}$. Significant differences $(p<0.04)$ were found between athlete and sedentary groups when $T_{\text {RSA1 }}$ and $T_{\text {RSA2 }}$ were expressed in terms of absolute and relative power and percentage of maximal aerobic power. In the sedentary group, $T_{R S A}$ and $T_{\text {RSA2 }}$ were 150.3 (18.7) W and $198.3(28.8) \mathrm{W}$, respectively, whereas in the athlete group $T_{R S A 1}$ and $T_{R S A 2}$ were 247.3 (32.8) W and 316.0 (28.8) W, respectively.

Conclusions: Dynamic analysis of $f_{\mathrm{RSA}}$ provides a useful tool for identifying ventilatory thresholds during graded and maximal exercise test in sedentary subjects and athletes.
T he spectral approach of heart period variability (HPV) has highlighted the fact that respiratory sinus arrhythmia (RSA) during exercise is the main mechanism regulating short term heart period (HP) fluctuations. ${ }^{1-3}$ RSA results from modulation of sinus node activity by breathing. Indeed, strong correlations have been found between the centred frequency of respiratory sinus arrhythmia $\left(f_{\mathrm{RSA}}\right)$ and respiratory frequency $\left(f_{\mathrm{R}}\right) .^{3-5}$ Classical spectral analysis requires stationarity of the studied signal. Consequently, studies of HPV and RSA during exercise are scarce. To overcome these limitations, time varying models have been developed which allow us to depict a signal divided into its instantaneous frequency and power components. During pyramidal exercises, the dynamic behaviour of $f_{\mathrm{RSA}}$ has been accurately extracted and strong links between $f_{\mathrm{RSA}}$ and $f_{\mathrm{R}}$ dynamic behaviours have been pointed out. ${ }^{6}$ This original approach to signal processing may be used in practice. For instance, Anosov et al ${ }^{4}$ have found that the dynamic behaviour of $f_{\mathrm{RSA}}$ extracted from HP series, recorded during a ramp load protocol, demonstrates significant changes in the region of the anaerobic threshold (AT). Previously, James et al showed that during graded exercise, the AT could be detected in healthy adults by $f_{\mathrm{R}}$ analysis. Moreover, ventilation $\left(\dot{V}_{\mathrm{I}}\right)$ time course analysis reveals two disproportionate increases in $\dot{\mathrm{V}}_{2},{ }^{7}$ defining the first and second ventilatory thresholds. These disproportionate increases are related to exercise induced acidosis compensation and are mainly linked to $f_{\mathrm{R}}$ increase. $^{89}$ Although disagreement exists, ${ }^{10}{ }^{11}$ ventilatory thresholds are closely related to lactate thresholds ${ }^{12-16}$ and could provide reliable indices of changes in response to endurance training or be useful when prescribing exercise training. ${ }^{17-19}$

As the two disproportionate increases in $\dot{V}_{\mathrm{I}}$ are explained by $f_{\mathrm{R}}$ disproportionate increases, analysis of $f_{\mathrm{RSA}}$ dynamic behaviour during a graded and maximal exercise test could reveal both the first and second ventilatory thresholds and provide practical applications as previously suggested. Such a method would be non-invasive and less expensive than the ventilatory flow and gas measurements required by ventilatory methods.

The first objective of this study was to use the signal processing method we previously developed ${ }^{6}$ to extract $f_{\mathrm{RSA}}$ from HP series recorded during graded and maximal exercise tests. Dynamic behaviours of $f_{\mathrm{RSA}}$ and ventilatory indices were then compared as regards exercise intensity in sedentary and athlete groups.

\section{METHODS}

Subjects

Fourteen sedentary healthy men (mean (SD) age: 24.5 (2.3) years) and 12 endurance athletes (age: 25.7 (2.8) years; $>12 \mathrm{~h}$ of training/week) (characteristics shown in table 1) participated in the study. All subjects were non-smokers and none was taking medication. Physical activity and consumption of alcohol and caffeinated beverages were prohibited $24 \mathrm{~h}$ before the exercise testing session. Written informed consent was obtained prior to participation and ethical approval was granted by the Local Ethics Committee.

\section{Experimental design}

Subjects performed a graded and maximal exercise test on a cycle ergometer (Ergomedic 824 E, Monark Exercise, Vansbro, Sweden) in a quiet room at a controlled temperature of $21^{\circ} \mathrm{C}$, at least $3 \mathrm{~h}$ after the last meal. In the sedentary and the athlete

Abbreviations: AT, anaerobic threshold; HP, heart period; HPV, heart period variability; RSA, respiratory sinus arrhythmia 
Table 1 Anthropometric and maximal ergometric characteristics of the subjects

\begin{tabular}{|c|c|c|c|c|c|}
\hline & \multicolumn{2}{|c|}{$\begin{array}{l}\text { Sedentary group } \\
(n=14)\end{array}$} & \multicolumn{2}{|c|}{$\begin{array}{l}\text { Athlete group } \\
(n=12)\end{array}$} & \multirow[b]{2}{*}{$\mathbf{p}$} \\
\hline & Mean & SD & Mean & SD & \\
\hline Age (years) & 24.5 & 2.3 & 25.7 & 2.8 & NS \\
\hline Height $(\mathrm{cm})$ & 173.4 & 9.1 & 183.8 & 5.6 & $* *$ \\
\hline Weight $(\mathrm{kg})$ & 68.3 & 9.9 & 81.4 & 8.5 & $* *$ \\
\hline$\dot{V}_{o_{2 m a x}}(\mathrm{ml} / \mathrm{min} / \mathrm{kg})$ & 44.7 & 4.6 & 56.4 & 9.3 & $* * *$ \\
\hline$W_{\max }(\mathrm{W})$ & 266.6 & 27.1 & 383.9 & 26.6 & $* * *$ \\
\hline $\mathrm{W}_{\max }(\mathrm{W} / \mathrm{kg})$ & 3.96 & 0.44 & 4.78 & 0.72 & $* *$ \\
\hline $\mathrm{HR}_{\max }(\mathrm{bpm})$ & 197.6 & 7.8 & 188.3 & 5.7 & ** \\
\hline
\end{tabular}

$H R$, heart rate; NS, not significant; $\dot{V}_{2 \text { max }}$, maximal oxygen uptake; $W_{\text {max }}$ maximal aerobic power.

Difference between groups: ${ }^{* *} \mathrm{p}<0.01 ;{ }^{* * *} \mathrm{p}<0.001$.

groups, the initial load was fixed at 75 and $150 \mathrm{~W}$, respectively, and increased by $37.5 \mathrm{~W}$ every $2 \mathrm{~min}$ until exhaustion. The pedalling rate was kept constant at $75 \mathrm{rev} / \mathrm{min}$.

Ventilatory indices and gas exchanges were measured using an automatic ergospirometer on a breath by breath basis (Metasys TR-M, Brainware, Toulon, France). Subjects breathed through a silicon facemask connected to a two-way non-rebreathing valve (Hans Rudolph, Kansas City, MO). Inspired and expired $\mathrm{O}_{2}$ and $\mathrm{CO}_{2}$ concentrations were measured using paramagnetic and infrared sensors, respectively. Averages every $10 \mathrm{~s}$ were then established for $\dot{\mathrm{V}}_{\mathrm{I}}$ (l/ min), $\mathrm{O}_{2}$ uptake $\left(\dot{\mathrm{V}}_{2}, \mathrm{l} / \mathrm{min}\right), \mathrm{CO}_{2}$ production $\left(\dot{\mathrm{V}}_{2}, \mathrm{l} / \mathrm{min}\right)$, respiratory ratio $(\mathrm{R})$, and ventilatory equivalents for $\mathrm{O}_{2}\left(\dot{\mathrm{V}}_{\mathrm{I}} /\right.$ $\left.\dot{\mathrm{V}} \mathrm{O}_{2}\right)$ and $\mathrm{CO}_{2}\left(\dot{\mathrm{V}}_{\mathrm{I}} / \dot{\mathrm{V}} \mathrm{CO}_{2}\right) . f_{\mathrm{R}}$ was calculated on a breath by breath basis. Before each test, the gas analysers were calibrated with gases of known composition and an accurate controlled volume syringe was used to adjust the pneumotachograph. During the exercise tests, a one lead ECG (Cardiocap II, Datex Engstrom, Helsinki, Finland) was recorded and digitised on line by a 12 bit analog-to-digital converter (DAS 1600, Keithley Instruments, Taunton, MA) at a sampling rate of $1000 \mathrm{~Hz}$, on a personal computer. Oxygen uptake was considered maximal $\left(\dot{\mathrm{V}}_{2 \max }\right)$ if three of the following criteria were met: levelling off of $\dot{\mathrm{V}}_{2}$ despite increasing load, R greater than 1.10 , and inability to maintain the fixed pedalling rate. The power corresponding to $\dot{\mathrm{V}}_{2 \max }$ defined the maximal aerobic power $\left(\mathrm{W}_{\max }\right)$.

\section{ECG preprocessing}

$\mathrm{R}$ wave peak occurrence was estimated using a threshold technique applied to the filtered and demodulated ECG signal.
HP series were visually inspected to ensure the absence of artefacts. In case of artefacts arising from a spurious $\mathrm{R}$ wave detection, the HP was restored by summing the two or more spuriously short periods. In cases of undetected $\mathrm{R}$ wave, the erroneous HP was replaced by using the two adjacent HP values. Artefacts did not exceed $1 \%$ of the total HP series. The first $20 \mathrm{~s}$ of exercise, which correspond to a marked HP decrease, were removed to limit sources of non-stationarity. In addition, the local mean HP was also removed using a polynomial approximation $p o(k)$ (order equal to 20) and a 100th order high pass finite impulse response filter was applied to the detrended HP series.

Since the stationarity conditions are not fulfilled under dynamic exercise, classical spectral analysis methods were replaced by a previously described method. ${ }^{60}$ Using this method, the dynamic behaviour of $f_{R S A}$ was extracted.

ECG preprocessing was performed using Matlab software 6.0 R12 (MathWorks, Natick, MA).

\section{Determination of ventilatory and RSA thresholds}

Ventilatory thresholds were determined from the time course curves of $\dot{\mathrm{V}}_{\mathrm{I}}, \dot{\mathrm{V}}_{\mathrm{I}} / \dot{\mathrm{V}}_{\mathrm{O}_{2}}$, and $\dot{\mathrm{V}}_{\mathrm{I}} / \dot{\mathrm{V}}_{\mathrm{CO}}$ by a first independent operator. $\mathrm{T}_{\mathrm{V} 1}$ corresponded to the last point before a first nonlinear increase in both $\dot{V}_{\mathrm{I}}$ and $\dot{\mathrm{V}}_{\mathrm{I}} / \dot{\mathrm{V}}_{2}$. $\mathrm{T}_{\mathrm{V} 2}$ corresponded to the last point before a second non-linear increase in both $\dot{V}_{I}$ and $\dot{\mathrm{V}}_{\mathrm{I}} / \dot{\mathrm{V}}_{2}$, accompanied by a non-linear increase in $\dot{\mathrm{V}}_{\mathrm{I}} / \dot{\mathrm{V}}_{\mathrm{CO}_{2}} \cdot{ }^{7}$ The $f_{\mathrm{RSA}}$ thresholds were determined from the time course curve of $f_{\mathrm{RSA}}$ by a second independent operator. The first $f_{\mathrm{RSA}}$ threshold $\left(\mathrm{T}_{\mathrm{RSAl}}\right)$ corresponded to the last point before a first non-linear increase in $f_{\mathrm{RSA}}$. The second $f_{\mathrm{RSA}}$ threshold ( $\left.\mathrm{T}_{\mathrm{RSA} 2}\right)$ corresponded to a second non-linear increase in $f_{\text {RSA }}$.

Thresholds were expressed in terms of absolute (W) and relative $(\mathrm{W} / \mathrm{kg})$ power and percentage of $\mathrm{W}_{\max }$.

\section{Statistical analysis}

Differences between the sedentary and athlete groups were tested using unpaired Student's $t$ test. Comparison and relationship between ventilatory and $f_{\mathrm{RSA}}$ thresholds were tested using paired Student's $t$ test and a linear regression analysis, respectively. Individual relationships between $f_{\mathrm{RSA}}$ and $f_{\mathrm{R}}$ were tested by calculating Pearson's r correlation coefficients. The mean (SD) of all individual correlation coefficients was then calculated. Statistical significance was set at $\mathrm{p}<0.05$. Results are means (SD). Statistical analysis was performed using Statistica software 5.5 (StatSoft, Tulsa, OK).

\section{RESULTS}

Athletes showed significantly higher values of $\dot{\mathrm{V}}_{2}$ and $\mathrm{W}_{\text {max }}$ when compared to sedentary subjects (see table 1 ).

Table 2 First and second thresholds obtained from $f_{\text {RSA }}$ and ventilatory indices, in sedentary and athlete groups

\begin{tabular}{|c|c|c|c|c|c|c|}
\hline & & \multicolumn{2}{|c|}{ Sedentary group ( $n=12$ ) } & \multicolumn{2}{|c|}{ Athlete group $(n=9)$} & \multirow[b]{2}{*}{$p$} \\
\hline & & Mean & SD & Mean & SD & \\
\hline \multirow[t]{4}{*}{ Absolute power (W) } & $\mathrm{T}_{\mathrm{RSA} 1}$ & 150.3 & 18.7 & 247.3 & 32.8 & *** \\
\hline & $\mathrm{T}_{\mathrm{V} 1}$ & 151.0 & 19.5 & 247.0 & 33.6 & *** \\
\hline & $\mathrm{T}_{\mathrm{RSA} 2}$ & 198.3 & 28.8 & 316.0 & 28.8 & *** \\
\hline & $\mathrm{T}_{\mathrm{V} 2}$ & 200.3 & 29.4 & 310.9 & 26.7 & *** \\
\hline \multirow[t]{4}{*}{ Relative power (W/kg) } & $\mathrm{T}_{\mathrm{RSA} 1}$ & 2.21 & 0.33 & 3.09 & 0.64 & *** \\
\hline & $\mathrm{T}_{\mathrm{V} 1}$ & 2.22 & 0.34 & 3.09 & 0.65 & *** \\
\hline & $\mathrm{T}_{\mathrm{RSA} 2}$ & 2.94 & 0.39 & 3.96 & 0.67 & *** \\
\hline & $\mathrm{T}_{\mathrm{V} 2}$ & 2.97 & 0.38 & 3.90 & 0.66 & *** \\
\hline \multirow[t]{4}{*}{$\% W_{\max }$} & $\mathrm{T}_{\mathrm{RSAl}}$ & 56.5 & 6.0 & 62.5 & 6.6 & * \\
\hline & $\mathrm{T}_{\mathrm{V} 1}$ & 56.8 & 6.7 & 62.3 & 5.7 & * \\
\hline & $\mathrm{T}_{\mathrm{RSA} 2}$ & 74.4 & 7.5 & 82.3 & 4.9 & ** \\
\hline & $\mathrm{T}_{\mathrm{V} 2}$ & 75.1 & 7.6 & 81.0 & 4.9 & * \\
\hline
\end{tabular}

$\% \mathrm{~W}_{\max }$, percentage of maximal aerobic power; $\mathrm{T}_{\mathrm{RSA}}$, first respiratory sinus arrhythmia frequency $\left(f_{\mathrm{RSA}}\right)$ threshold; $\mathrm{T}_{\mathrm{RSA} 2}$, second $f_{\mathrm{RSA}}$ threshold; $\mathrm{T}_{\mathrm{V} 1}$, first ventilatory threshold; $\mathrm{T}_{\mathrm{V} 2}$, second ventilatory threshold.

Difference between groups: ${ }^{*} \mathrm{p}<0.05 ;{ }^{* *} \mathrm{p}<0.01 ;{ }^{* * *} \mathrm{p}<0.001$ 


\section{$f_{\mathrm{RSA}}$ extraction}

A conspicuous high frequency oscillation synchronous with $f_{R}$ was found in all ECG recordings, clearly indicating the persistence of RSA over the entire graded and maximal exercise protocol. The dynamic evolution of $f_{\text {RSA }}$ was accurately extracted from the HP series and $f_{\text {RSA }}$ positively correlated $(\mathrm{r}=0.96(0.03), \mathrm{p}<0.01)$ with $f_{\mathrm{R}}($ fig $\mathrm{l})$.

\section{$f_{\mathrm{RSA}}$ dynamic behaviour}

Two non-linear increases were observed in $f_{\mathrm{RSA}}$ in 21 of the 26 subjects. These non-linear increases coincided with $\mathrm{T}_{\mathrm{Vl}}$ and $\mathrm{T}_{\mathrm{V} 2}$, respectively (see fig 2 ) and no statistical difference was observed between $\mathrm{T}_{\mathrm{RSAl}}$ and $\mathrm{T}_{\mathrm{Vl}}$ (absolute power: $\mathrm{p}=0.98$; relative power: $\mathrm{p}=0.90$; percentage of $\mathrm{W}_{\text {max }}$ : $\mathrm{p}=0.91$ ) and $\mathrm{T}_{\mathrm{RSA} 2}$ and $\mathrm{T}_{\mathrm{V} 2}$ (absolute power: $\mathrm{p}=0.57$; relative power: $\mathrm{p}=0.79$; percentage of $\mathrm{W}_{\max }: \mathrm{p}=0.78$ ). Power values and percentages of $\mathrm{W}_{\max }$ at $\mathrm{T}_{\mathrm{RSAl}}, \mathrm{T}_{\mathrm{RSA} 2}, \mathrm{~T}_{\mathrm{V} 1}$, and $\mathrm{T}_{\mathrm{V} 2}$ are presented in table 2 . When expressed as absolute or relative power and percentage of $\mathrm{W}_{\max }, \mathrm{T}_{\mathrm{RSAl}}, \mathrm{T}_{\mathrm{RSA} 2}, \mathrm{~T}_{\mathrm{V} 1}$, and $\mathrm{T}_{\mathrm{V} 2}$ were significantly higher in athletes than in their sedentary peers. Linear regression analysis showed high correlation between $\mathrm{T}_{\mathrm{RSAl}}$ and $\mathrm{T}_{\mathrm{V} 1}$ (absolute power: $\mathrm{r}=0.99$, $\mathrm{p}<0.001$ (fig 3); relative power: $\mathrm{r}=0.99, \mathrm{p}<0.001$; percentage of $\mathrm{W}_{\text {max }}: \mathrm{r}=0.95, \mathrm{p}<0.001$ ) and $\mathrm{T}_{\mathrm{RSA} 2}$ and $\mathrm{T}_{\mathrm{V} 2}$ (absolute power: $r=0.99, p<0.001$ (fig 3); relative power: $r=0.99$, $\mathrm{p}<0.001$; percentage of $\mathrm{W}_{\text {max }}: \mathrm{r}=0.96, \mathrm{p}<0.001$ ).

In the five remaining subjects (three athletes and two sedentary subjects) only one non-linear increase was clearly identifiable and occurred close to $\mathrm{T}_{\mathrm{V} 2}$ (fig 4).

\section{DISCUSSION}

To assess HPV and RSA during non-stationary exercise conditions, we developed and validated an original method. ${ }^{6}$ In the present study, this method was used to process the cardiac electrical signal during a maximal and graded exercise test.

Using our original approach, the dynamic pattern of $f_{\mathrm{RSA}}$ was accurately extracted from R-R interval series; RSA and breathing have been shown to develop dynamically at the same frequency. This result confirms previous findings ${ }^{3-5}$ which showed that during exercise, heart rate is modulated by breathing at the $f_{\mathrm{R}}$. When $f_{\mathrm{RSA}}$ was considered, we were able to point out two successive non-linear increases in $81 \%$ of our population. First, we observed that $\mathrm{T}_{\mathrm{RSAl}}$ was closely related to $T_{\mathrm{Vl}}$. This finding is consistent with those of Anosov et $a l^{4}$ who reported that significant changes in the behaviour of $f_{\mathrm{RSA}}$ occurred in the region of the AT. As the $f_{\mathrm{RSA}}$ pattern is

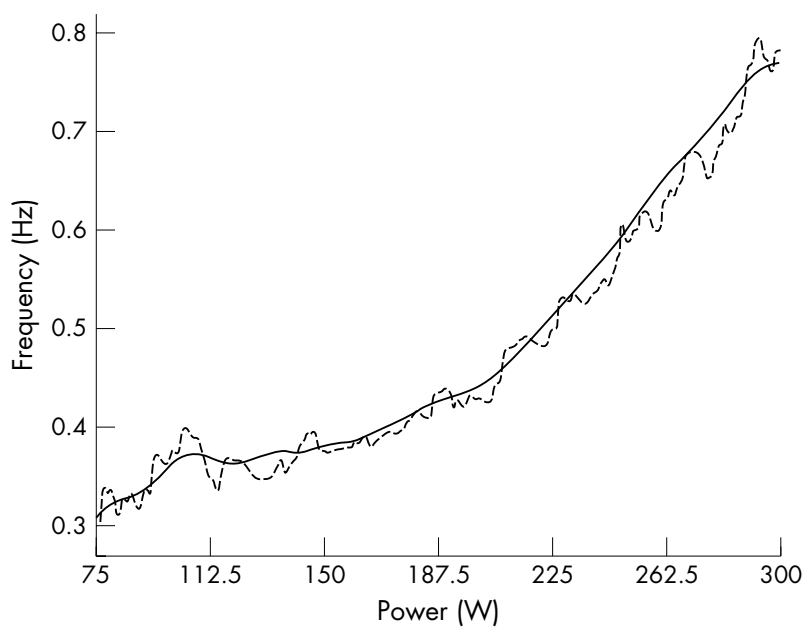

Figure 1 Representation of the dynamic behaviour of RSA frequency ( $f_{R S A}$, solid line) and respiratory frequency $\left(f_{R}\right.$, dashed line) recorded in one subject during graded and maximal exercise test.
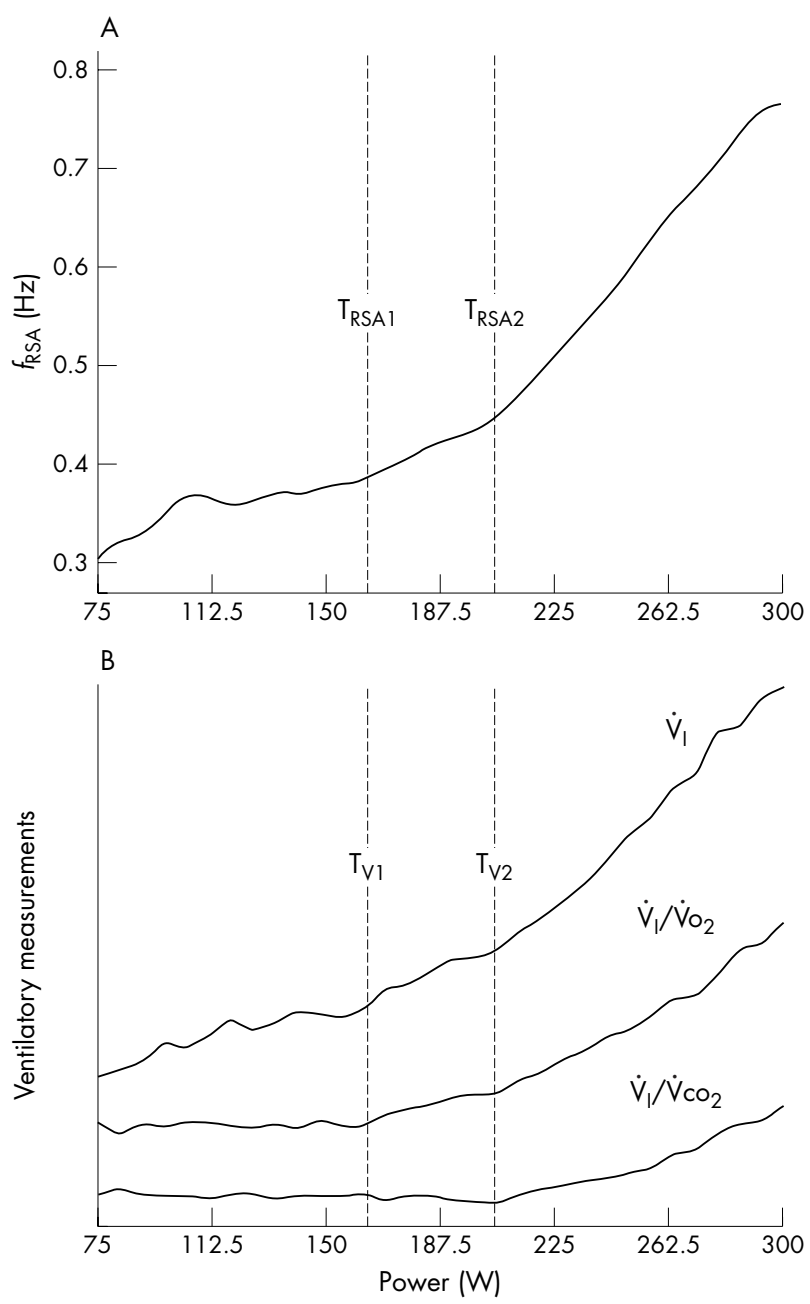

Figure 2 Example of threshold determination using $f_{R S A}(A)$ and ventilatory (B) methods. RSA $_{\text {, }}$ respiratory sinus arrhythmia frequency; $T_{R S A}$, first $f_{R S A}$ threshold; $T_{R S A 2}$, second $f_{R S A}$ threshold; $T_{V 1}$, first ventilatory threshold; $T_{V 2}$, second ventilatory threshold; $V_{1}$, ventilation; $\dot{\mathrm{V}}_{1} / \mathrm{V}_{2}$, ventilatory equivalents for $\mathrm{O}_{2} ; \dot{\mathrm{V}}_{1} / \mathrm{V}_{\mathrm{CO}_{2}}$, ventilatory equivalents for $\mathrm{CO}_{2}$.

closely linked to $f_{\mathrm{R}}$, we could state that the first disproportionate increase in $\dot{V}_{I}$ observed at $\mathrm{T}_{\mathrm{VI}}$ is mainly induced by an increase in $f_{\mathrm{R}}$. This is confirmed by the study of James et al ${ }^{8}$ who concluded that the first ventilatory threshold (referred as the AT in their study) could be detected by $f_{\mathrm{R}}$ analysis.

Second, we observed that $\mathrm{T}_{\mathrm{RSA} 2}$ was closely related to $\mathrm{T}_{\mathrm{V} 2}$, suggesting that the second disproportionate increase in $\dot{\mathrm{V}}_{\mathrm{I}}$ is again related to $f_{\mathrm{R}}$ increase. It has been reported that $\mathrm{T}_{\mathrm{V} 2}$ determines the workload before a marked fall in capillary pH. ${ }^{7}$ This exercise induced metabolic acidosis then causes ventilation increase through an increase in $f_{\mathrm{R}}{ }^{9}{ }^{9}$

The concept of ventilatory thresholds is closely linked in the literature to the concept of AT. AT is defined as the intensity of exercise, involving a large muscle mass, above which the oxidative metabolism cannot account for all the required energy and the anaerobic contribution to energy demand increases. ${ }^{21}$ Numerous studies have been conducted to detect one or two thresholds in metabolic (lactate for instance) or ventilatory indices time course curves. This diversity in methods of detection as well as lack of consensus on the theoretical basis have led to confusion and misinterpretation (see Bosquet $e t$ al ${ }^{19}$ and Svedahl and MacIntosh ${ }^{21}$ for reviews). Using blood lactate concentration is probably the most direct and reliable method to detect the AT. ${ }^{19}$ However, this method is invasive and requires 

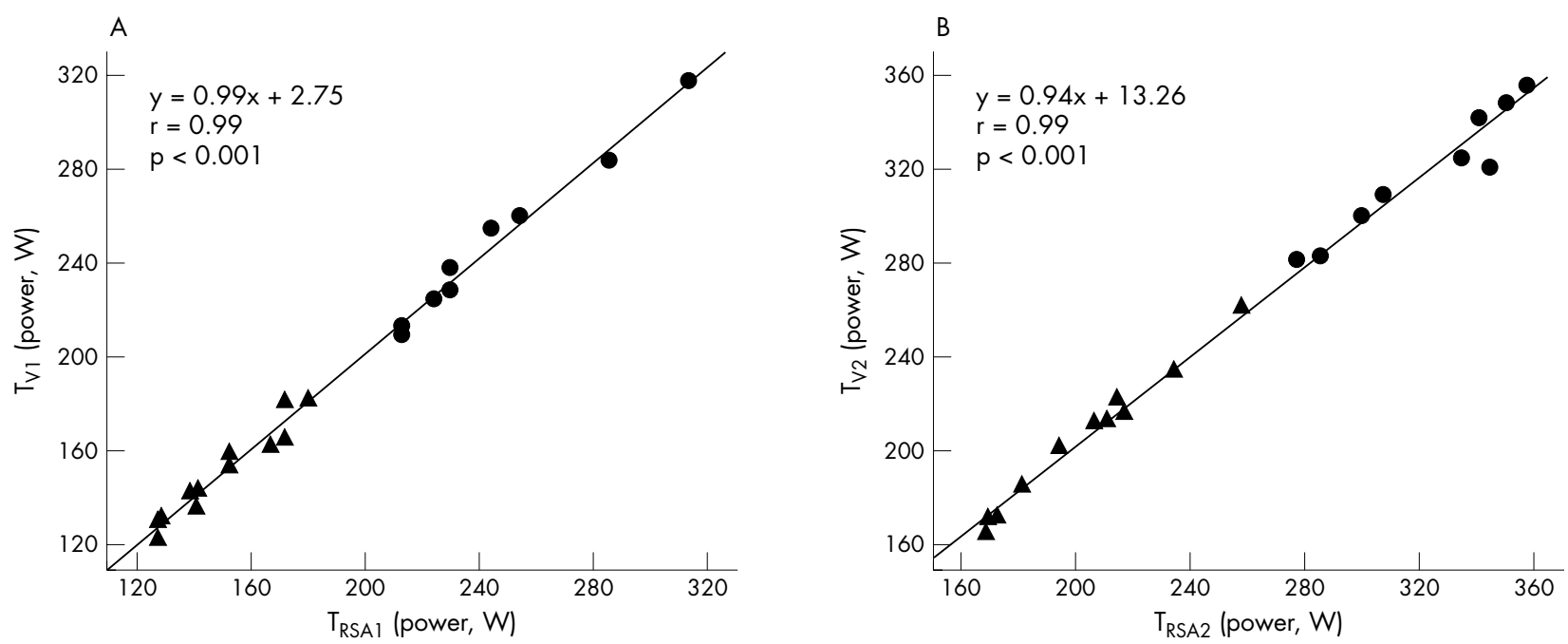

Figure 3 Relationships between absolute power measured at $T_{R S A 1}$ and $T_{V 1}(A)$ and $T_{R S A 2}$ and $T_{V 2}(B)$. Solid lines represent the regression lines. - Athlete group; $\boldsymbol{\Delta}$ sedentary group. $\mathrm{T}_{\mathrm{RSA} 1}$, first respiratory sinus arrhythmia frequency $\left(f_{\mathrm{RSA}}\right)$ threshold; $\mathrm{T}_{\mathrm{RSA}}$, second $f_{\mathrm{RSA}}$ threshold; $\mathrm{T}_{\mathrm{V} 1}$, first ventilatory threshold; $\mathrm{T}_{\mathrm{V} 2}$, second ventilatory threshold.

frequent blood sampling which is uncomfortable during continuous exercise. The indirect technique using ventilatory indices could be thus preferable. Indeed, although disagreement exists, ${ }^{10}{ }^{11}$ ventilatory thresholds are known to be closely related to lactate thresholds. ${ }^{12-16}$ Ventilatory threshold detection is
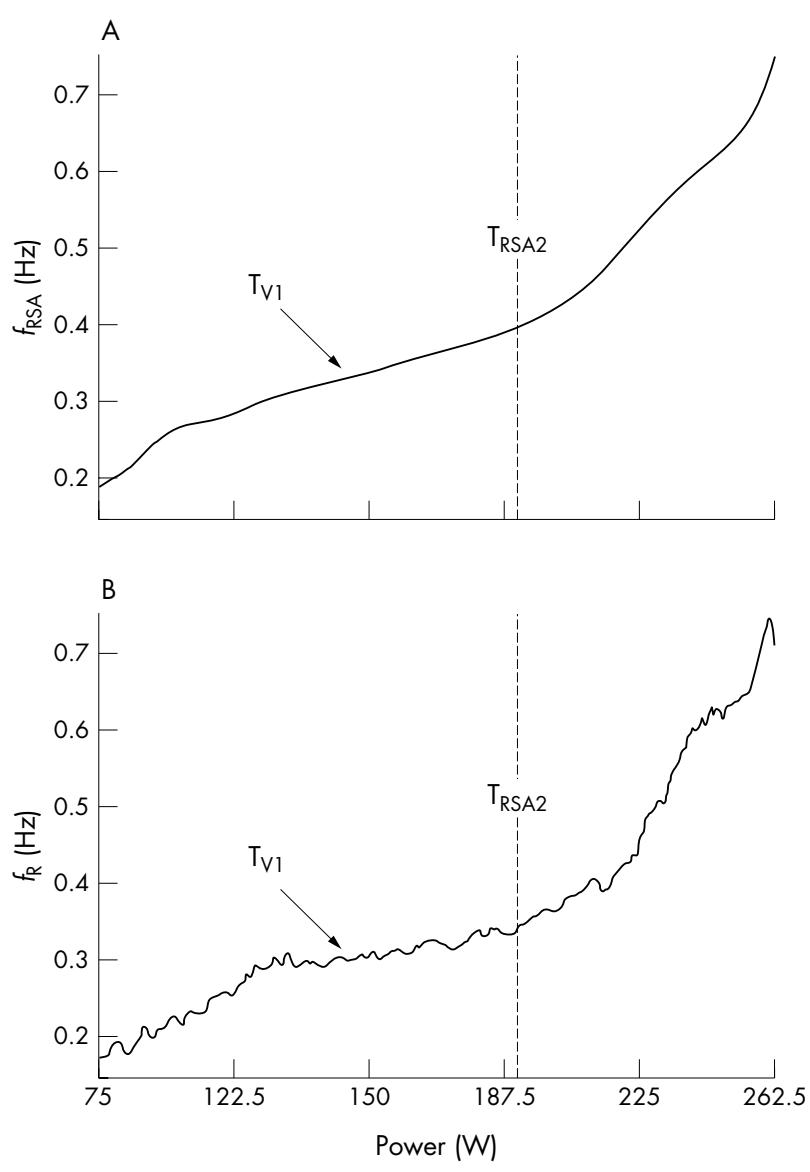

Figure 4 Example of lack of clear change in $f_{R S A}(A)$ and $f_{R}(B)$ in the region of $T_{V 1} \cdot f_{R}$, respiratory frequency; $f_{R S A}$, respiratory sinus arrhythmia frequency; $T_{R S A}$, second $f_{\text {RSA }}$ threshold; $T_{V 1}$, first ventilatory threshold. usually based on assessment of successive disproportionate increases in $\dot{V}_{\mathrm{I}}$, and $f_{\mathrm{R}}$ is known to play a major role in these increases. ${ }^{8}{ }^{9}$ It is also known that heart activity is modulated by breathing at the $f_{\mathrm{R}}$ and this modulation represents the RSA which is vagally mediated at rest. ${ }^{22-24}$ Although cardiac vagal tone is totally abolished over $\pm 60 \%$ of $\dot{\mathrm{V}}_{2 \max }{ }^{25}$ to adapt heart activity to cell metabolic demand, ${ }^{26}{ }^{27}$ RSA was retrieved over our entire exercise test. This finding confirms that RSA persistence at intense exercise could be related to enhancement of a non-neural mechanism in response to $\dot{V}_{I}$ increase. Indeed, changes in thoracic pressure induced by breathing influence filling of the right ventricle. ${ }^{29}$ Increased right ventricle filling during inspiration consequently increases transmural pressure and stretches the sinus node, thus activating positive chronotropic response via mechanosensitive $\mathrm{Cl}^{-}$channels. ${ }^{30} 31$

Thus, using $f_{\text {RSA }}$ to detect ventilatory thresholds has the advantages of being non-invasive and cheap and may have field application in ambulatory heart rate monitors. Moreover, this technique appears to be reliable in most athletes and sedentary subjects. $f_{\mathrm{RSA}}$ thresholds of athletes were detected at higher values than those of their sedentary peers, whatever the mode of expression, confirming that the AT is significantly improved with endurance training. ${ }^{32} 33$ Thus, this $f_{\text {RSA }}$ method could be used for the determination of human ventilatory thresholds over a broad range of physical abilities. However, in $19 \%$ of our population only one increase close to $T_{\mathrm{V} 2}$ was clearly identifiable in $f_{\mathrm{RSA}}$, whereas two ventilatory thresholds were detected. As $\dot{V}_{I}$ is the product of $f_{R}$ and $V_{T}$, it could be expected that the first non-linear increases in $\dot{\mathrm{V}}_{\mathrm{I}}$ and $\dot{\mathrm{V}}_{\mathrm{I}} / \dot{\mathrm{V}}_{\mathrm{O}_{2}}$ were mainly related to $\mathrm{V}_{\mathrm{T}}$ increase. Indeed, as shown in fig 4 , no clear change in $f_{\mathrm{R}}$ was observed around absolute power corresponding to $\mathrm{T}_{\mathrm{Vl}}$.

Visual detection of both ventilatory and $f_{\text {RSA }}$ thresholds can lead to subjective results and may represent a methodological limitation of our study design. Indeed, it has been shown that different evaluators can choose different ventilatory thresholds from the same data. ${ }^{34}$ However, reliability of the ventilatory method is known to be enhanced when test conditions are kept constant and evaluators are experienced, ${ }^{21}$ which was the case in our study. Detection of ventilatory threshold is known to be dependant both on stage duration and load increase in graded exercise..$^{35}$ As no exercise protocol test seems consensual, the standard protocol test used in our laboratory was thus preferred. 


\section{What is already known on this topic}

Respiratory sinus arrhythmia results from modulation of sinus node activity by breathing and during exercise is the main mechanism regulating short term heart period fluctuations. Strong correlations have been found between the centred frequency of respiratory sinus arrhythmia and respiratory frequency.

We have shown that, in most of our subjects, two successive non-linear increases are observed in $f_{\mathrm{RSA}}$. These thresholds are closely related to the first and second ventilatory thresholds, respectively. Thus, the method we developed provides a useful tool for identifying the ventilatory thresholds during graded and maximal exercise test in athletes and sedentary subjects as well as for assessing endurance levels. The next step could be to process HP series recorded during an adapted field test using modern heart rate monitors and time varying modelling.

\section{ACKNOWLEDGEMENTS}

We thank the Brainware Company for their technical support.

\section{Authors' affiliations}

G Blain, S Bermon, Département Ergonomie Sportive et Performances, Universite de Toulon-Var, Nice, France

O Meste, Laboratoire I3S, Universite de Nice Sophia-Antipolis, Nice, France

T Bouchard, Universite de Nice Sophia-Antipolis, Nice, France

Competing interests: none declared

\section{REFERENCES}

1 Perini R, Orizio C, Baselli G, et al. The influence of exercise intensity on the power spectrum of heart rate variability. Eur J Appl Physiol Occup Physiol 1990:61:143-8.

2 Perini R, Milesi S, Fisher NM, et al. Heart rate variability during dynamic exercise in elderly males and females. Eur J Appl Physiol 2000;82:8-15.

3 Bernardi L, Keller F, Sanders M, et al. Respiratory sinus arrhythmia in the denervated human heart. J Appl Physiol 1989;67:1447-55.

4 Anosov O, Patzak A, Kononovich Y, et al. High-frequency oscillations of the heart rate during ramp load reflect the human anaerobic threshold. Eur J Appl Physiol 2000;83:388-94.

5 Casadei B, Moon J, Johnston J, et al. Is respiratory sinus arrhythmia a good index of cardiac vagal tone in exercise? J Appl Physiol 1996:81:556-64.

6 Meste O, Blain G, Bermon S. Analysis of the respiratory and cardiac systems coupling in pyramidal exercise using a time-varying model. In: Computers in cardiology. Memphis, TN: IEEE Computer Press, 2002:429-32.

7 Reinhard U, Muller PH, Schmulling RM. Determination of anaerobic threshold by the ventilation equivalent in normal individuals. Respiration 1979:38:36-42

8 James NW, Adams GM, Wilson AF. Determination of anaerobic threshold by ventilatory frequency. Int J Sports Med 1989;10:192-6.

9 Wasserman K. Breathing during exercise. N Engl J Med 1978;298:780-5.

10 Hughes EF, Turner SC, Brooks GA. Effects of glycogen depletion and pedaling speed on "anaerobic threshold". J Appl Physiol 1982;52:1598-607.

11 Green HJ, Hughson RL, Orr GW, et al. Anaerobic threshold, blood lactate, and muscle metabolites in progressive exercise. J Appl Physiol 1983;54:1032-8.

12 Aunola S, Rusko H. Aerobic and anaerobic thresholds determined from venous lactate or from ventilation and gas exchange in relation to muscle fiber composition. Int J Sports Med 1986;7:161-6.

13 Davis JA, Vodak P, Wilmore JH, et al. Anaerobic threshold and maximal aerobic power for three modes of exercise. J Appl Physiol 1976;41:544-50.

14 Yoshida T, Nagata A, Muro M, et al. The validity of anaerobic threshold determination by a Douglas bag method compared with arterial blood lactate concentration. Eur J Appl Physiol Occup Physiol 1981;46:423-30.

15 Caiozzo VJ, Davis JA, Ellis JF, et al. A comparison of gas exchange indices used to detect the anaerobic threshold. J Appl Physiol 1982;53:1 184-9.

16 Burke J, Thayer R, Belcamino M. Comparison of effects of two interval-training programmes on lactate and ventilatory thresholds. Br J Sports Med 1994;28:18-21.

17 Kindermann W, Simon G, Keul J. The significance of the aerobic-anaerobic transition for the determination of work load intensities during endurance training. Eur J Appl Physiol Occup Physiol 1979;42:25-34.

18 Farrell PA, Wilmore JH, Coyle EF, et al. Plasma lactate accumulation and distance running performance. Med Sci Sports 1979;1 1:338-44.

19 Bosquet L, Leger L, Legros P. Methods to determine aerobic endurance. Sports Med 2002;32:675-700
What this study adds

Two successive non-linear increases observed in respiratory sinus arrhythmia frequency are closely related to the first and second ventilatory thresholds, respectively. We have developed a useful method for identifying the ventilatory thresholds during graded and maximal exercise test in athletes and sedentary subjects as well as for assessing endurance levels.

20 Meste O, Blain G, Bermon S. Some considerations on the IPFM model for the heart rate variability analysis. In: Computers in Cardiology, 2003. Thessaloniki, Greece: IEEE Computer Society Press, 2003:709-12.

21 Svedahl K, Maclntosh BR. Anaerobic threshold: the concept and methods of measurement. Can J Appl Physiol 2003;28:299-323.

22 Akselrod S, Gordon D, Ubel FA, et al. Power spectrum analysis of heart rate fluctuation: a quantitative probe of beat-to-beat cardiovascular control. Science 1981;213:220-2

23 Malliani A, Pagani M, Lombardi F, et al. Cardiovascular neural regulation explored in the frequency domain. Circulation 1991;84:482-92.

24 Pomeranz B, Macaulay RJ, Caudill MA, et al. Assessment of autonomic function in humans by heart rate spectral analysis. Am J Physiol 1985;248:H151-3

25 Warren JH, Jaffe RS, Wraa CE, et al. Effect of autonomic blockade on power spectrum of heart rate variability during exercise. Am J Physiol 1997;273:R495-502.

26 Ekblom B, Goldbarg AN, Kilbom A, et al. Effects of atropine and propranolol on the oxygen transport system during exercise in man. Scand J Clin Lab Invest 1972;30:35-42.

27 Robinson BF, Epstein SE, Beiser GD, et al. Control of heart rate by the autonomic nervous system. Studies in man on the interrelation between baroreceptor mechanisms and exercise. Circ Res 1966;19:400-11.

28 Blain G, Meste O, Bermon S. Influences of breathing patterns on respiratory sinus arrhythmia in humans during exercise. Am J Physiol Heart Circ Physiol 2005;288(2): H887-95.

29 Cournand A, Motley HL, Werko L, et al. Physiological studies of the effects of intermittent positive pressure breathing on cardiac output in man. Am J Physiol 1948; 152:162-74.

30 Arai A, Kodama I, Toyama J. Roles of $\mathrm{Cl}^{-}$channels and $\mathrm{Ca}^{2+}$ mobilization in stretch-induced increase of SA node pacemaker activity. Am J Physiol 1996;270: $\mathrm{H} 1726-35$

31 Hagiwara N, Masuda H, Shoda $M$, et al. Stretch-activated anion currents of rabbit cardiac myocytes. J Physiol 1992;456:285-302.

32 Rowbottom DG, Keast D, Garcia-Webb P, et al. Training adaptation and biological changes among well-trained male triathletes. Med Sci Sports Exerc 1997;29:1233-9.

33 Foster C, Fitzgerald DJ, Spatz P. Stability of the blood lactate-heart rate relationship in competitive athletes. Med Sci Sports Exerc 1999;31:578-82.

34 Yeh MP, Gardner RM, Adams TD, et al. "Anaerobic threshold": problems of determination and validation. J Appl Physiol 1983;55:1178-86.

35 Kang J, Chaloupka EC, Mastrangelo MA, et al. Physiological comparisons among three maximal treadmill exercise protocols in trained and untrained individuals. Eur J Appl Physiol 2001;84:291-5.

\section{COMMENTARY}

During the past 20 years, very many studies have indicated that parameters measured during submaximal exercise may be better markers of endurance performance than $\mathrm{Vo}_{2 \text { max }}$, the anaerobic (or ventilatory) and lactate thresholds being useful parameters to evaluate functional capability in various types of endurance performance. Both gas analysis and ventilatory flow measurements, as well as blood lactate determinations, can be used to estimate the anaerobic threshold as a predictor of endurance capacity. A procedure that would be simple, relatively inexpensive, and non-invasive would be welcome. Procedures based on maximal heart rate (or a percentage of it) are simple but not reliable. Thus, the determination of ventilatory thresholds by time varying analysis of respiratory sinus arrhythmia, as proposed in this paper, appears to be quite promising, providing that it can be used with data obtained by ambulatory heart rate monitors.

Physiological Sciences II, Universitat de Barcelona, $\begin{array}{r}\text { Ramon Segura } \\ \text { Barcelona, Spain; } \\ \text { rasegura@ub.edu }\end{array}$ 